\title{
Research on Guidance Instrumentation Error Separation Method of Platform Inertial Navigation System
}

\author{
Hongjie Zhang ${ }^{1, a}$, Shifeng Zhang, ${ }^{1, b *}$ \\ ${ }^{1}$ College of Aerospace Science and Engineering, National University of Defense Technology, Deya Road, \\ Changsha, China \\ ahitzhanghongjie@163.com, bzhang_shifeng@hotmail.com, \\ *corresponding author
}

Keywords: guidance instrumentation error, error separation, wavelet, RELS.

\begin{abstract}
Aiming at the existing problems of the current methods in the area of guidance instrumentation error separation, such as the multicollinearity impact, the large noise effect and the low accuracy of separation results, a brand new algorithm is proposed. Firstly, basing on the advantage of wavelet analysis on disposing signals, data de-nosing is carried out; secondly, using the feature of recursive extended least square (RELS) method that the noise sequence is considered as the identification object to separate the instrumentation error. The simulation results show that, the proposed method not only can decrease the impact of noise but also can improve the accuracy of the estimation of error coefficients.
\end{abstract}

\section{Introduction}

Briefly speaking, guidance instrumentation error is the impact miss caused by the imperfection of the guidance equipment. The existing data shows that, the error of inertial platform is the primary error source which can account for about 70\% - 80\% [1] of all the instrumentation error. The instrumentation error separation is the basement to guarantee the missile hit accuracy. Comparing with the ground calibration test, the driving level of each error coefficient is uneven during the flight of missiles. So how to choose an appropriate method to separate the instrumentation error is of vital importance.

The main traditional methods include the least square method, the principal component analysis and so on. These methods have the shortage that can be seriously affected by the multicollinearity. The separation results are not good enough. To improve the accuracy of separation, the wavelet analysis and the recursive extended least square method are applied to the instrumentation error separation.

The wavelet analysis is widely used in the field of engineering after being explained from the engineering standpoint by Mallat [2] and Daubechies in the 1990s. The wavelet analysis is an ideal signal analysis tool [3], especially in the de-nosing aspect. Huang [4] applied the wavelet analysis to the real-time de-nosing of the fibre optic gyro of the floating inertial platform. The effect is better compared with the least mean square adaptive filtering.

The RELS can be regarded as the simple extension of the regular least square and is based on the recursive least square (RLS). The noise sequence is considered as the identification object in the RELS [5]. And as a result, the dimensions of the parameter vector and the data vector are extended. RELS has a very good convergence [6].

On the basis of the wavelet analysis and RELS, a brand new algorithm to separate the instrumentation error is proposed in this paper.

\section{Model of Instrumentation Error Separation}

The installation of the accelerometers and gyros on the platform is shown in the following figure: 


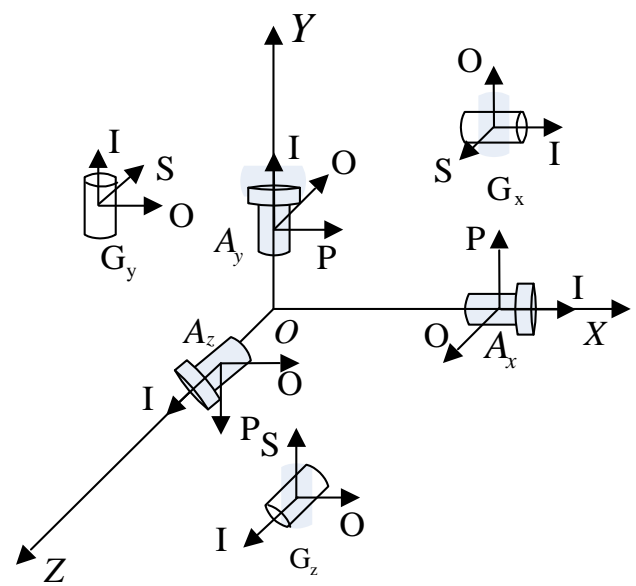

Figure 1 The installation of the IMU

The system model of the instrumentation error separation is built on the basis of the relationship between the instrumentation error coefficient and the velocity error which is the difference between the telemetric data and tracking data. The error of the telemetric data is small enough to ignore. So the telemetric data is considered as error-free.

Gyro models adopted in this paper are shown as:

$$
\left\{\begin{array}{l}
\dot{\varphi}_{x}=K_{g 0 x}+K_{g 1 x} \dot{W}_{p x}+K_{g 1 x y} \dot{W}_{p y}+K_{g 1 x z} \dot{W}_{p z}+K_{g 2 x} \dot{W}_{p x}^{2} \\
\dot{\varphi}_{y}=K_{g 0 y}+K_{g 1 y} \dot{W}_{p y}+K_{g 1 y z} \dot{W}_{p z}+K_{g 1 y x} \dot{W}_{p x}+K_{g 2 y} \dot{W}_{p y}^{2} \\
\dot{\varphi}_{z}=K_{g 0 z}+K_{g 1 z} \dot{W}_{p z}+K_{g 1 z x} \dot{W}_{p x}+K_{g 1 z y} \dot{W}_{p y}+K_{g 2 z} \dot{W}_{p z}^{2}
\end{array}\right.
$$

Where:

$\dot{\varphi}_{x}, \dot{\varphi}_{y}, \dot{\varphi}_{z}$ : The drift angular velocity of the $X$-gyro 、 $Y$-gyro and Z-gyro;

$K_{g 0 x} 、 K_{g 0 y} 、 K_{g 0 z}$ : The bias of the gyros along the $x, y, z$ axis;

$K_{g 1 x} 、 K_{g 1 x y} 、 K_{g 1 x z}$ : The first-order terms of the $X$-gyro along its input axis 、 output axis and spin axis;

$K_{g 1 y} 、 K_{g 1 y z}, K_{g 1 y x}$ : The first-order terms of the $Y$-gyro along its input axis spin axis and output axis;

$K_{g 1 z} 、 K_{g 1 z x} 、 K_{g 1 z y}$ : The first-order terms of the Z-gyro along its input axis、spin axis and output axis;

$K_{g 2 x} 、 K_{g 2 y} 、 K_{g 2 z}$ : The second-order terms of the gyros;

$\dot{W}_{p x} 、 \dot{W}_{p y} 、 \dot{W}_{p z}$ : The specific force measured by the accelerometer on the platform.

Put Eq. (1) into matrix form:

$\dot{\boldsymbol{\varphi}}=\boldsymbol{S}_{g} \boldsymbol{D}_{g}$

Where:

$\boldsymbol{S}_{g}=\left[\begin{array}{ccccccc}1 & \dot{W}_{p x} & \dot{W}_{p y} & \dot{W}_{p z} & \dot{W}_{p x}^{2} & \boldsymbol{0}_{1 \times 5} & \boldsymbol{0}_{1 \times 5} \\ \boldsymbol{0}_{1 \times 5} & 1 & \dot{W}_{p y} & \dot{W}_{p z} & \dot{W}_{p x} & \dot{W}_{p y}^{2} & \boldsymbol{0}_{1 \times 5} \\ \boldsymbol{0}_{1 \times 5} & \boldsymbol{0}_{1 \times 5} & 1 & \dot{W}_{p z} & \dot{W}_{p x} & \dot{W}_{p y} & \dot{W}_{p z}^{2}\end{array}\right]$;

$\boldsymbol{0}_{1 \times 5}$ : The 1-by-5 matrix of which every element is 0 ;

$$
\begin{aligned}
& \boldsymbol{D}_{g x}=\left[\begin{array}{lllll}
K_{g 0 x} & K_{g 11 x} & K_{g 12 x} & K_{g 13 x} & K_{g 2 x}
\end{array}\right]^{T}, \quad \boldsymbol{D}_{g y}=\left[\begin{array}{llllll}
K_{g 0 y} & K_{g 11 y} & K_{g 12 y} & K_{g 13 y} & K_{g 2 y}
\end{array}\right]^{T} \\
& \boldsymbol{D}_{g z}=\left[\begin{array}{lllllll}
K_{g 0 z} & K_{g 11 z} & K_{g 12 z} & K_{g 13 z} & K_{g 2 z}
\end{array}\right]^{T}, \boldsymbol{D}=\left[\begin{array}{lllll}
\boldsymbol{D}_{g x} & \boldsymbol{D}_{g y} & \boldsymbol{D}_{g z}
\end{array}\right]^{\mathrm{T}}
\end{aligned}
$$

Accelerometer models adopted in this paper are shown as: 


$$
\left\{\begin{array}{l}
\Delta_{x}=K_{a 0 x}+K_{a 1 x} \dot{W}_{p x}+K_{a 1 x y} \dot{W}_{p y}+K_{a 1 x z} \dot{W}_{p z}+K_{a 2 x} \dot{W}_{p x}^{2}+K_{a 2 x y} \dot{W}_{p x} \dot{W}_{p y}+K_{a 2 x z} \dot{W}_{p x} \dot{W}_{p z} \\
\Delta_{y}=K_{a 0 y}+K_{a 1 y} \dot{W}_{p y}+K_{a 1 y z} \dot{W}_{p z}+K_{a 1 y x} \dot{W}_{p x}+K_{a 2 y} \dot{W}_{p y}^{2}+K_{a 2 y z} \dot{W}_{p y} \dot{W}_{p z}+K_{a 2 y x} \dot{W}_{p y} \dot{W}_{p x} \\
\Delta_{z}=K_{a 0 z}+K_{a 1 z} \dot{W}_{p z}+K_{a 1 z x} \dot{W}_{p x}+K_{a 1 z y} \dot{W}_{p y}+K_{a 2 z} \dot{W}_{p z}^{2}+K_{a 2 z x} \dot{W}_{p z} \dot{W}_{p x}+K_{a 2 z y} \dot{W}_{p z} \dot{W}_{p y}
\end{array}\right.
$$

Where:

$\Delta_{x} 、 \Delta_{y} 、 \Delta_{z}$ : The measuring error of accelerometers;

$K_{a 0 x} 、 K_{a 0 y} 、 K_{a 0 z}$ : The scale factors of the accelerometers;

$K_{a 1 x y} 、 K_{a 1 x z}$ : The influence coefficients caused by the non-perpendicularity of $\mathrm{y} 、 \mathrm{z}$ axis to $\mathrm{x}$ axis;

$K_{a 1 y z} 、 K_{a 1 y x}$ : The influence coefficients caused by the non-perpendicularity of $\mathrm{x} 、 \mathrm{z}$ axis to $\mathrm{y}$ axis;

$K_{a 1 z x} 、 K_{a 1 z y}$ : The influence coefficients caused by the non-perpendicularity of $\mathrm{x} 、 \mathrm{y}$ axis to $\mathrm{z}$ axis;

$K_{a 2 x} 、 K_{a 2 y} 、 K_{a 2 z}$ : The second-order terms of the accelerometers;

$K_{a 2 x y} 、 K_{a 2 x z} 、 K_{a 2 y z} 、 K_{a 2 y x} 、 K_{a 2 z x} 、 K_{a 2 z y}$ : The cross-order terms of the accelerometers.

Put Eq. (3) into matrix form:

$\boldsymbol{\Delta}=\boldsymbol{S}_{a} \boldsymbol{D}_{a}$

Where:

$\boldsymbol{S}_{a}=\left[\begin{array}{ccccccccc}1 & \dot{W}_{p x} & \dot{W}_{p y} & \dot{W}_{p z} & \dot{W}_{p x}^{2} & \dot{W}_{p x} \dot{W}_{p y} & \dot{W}_{p x} \dot{W}_{p z} & \boldsymbol{0}_{1 \times 7} & \boldsymbol{0}_{1 \times 7} \\ \boldsymbol{0}_{1 \times 7} & 1 & \dot{W}_{p y} & \dot{W}_{p z} & \dot{W}_{p x} & \dot{W}_{p y}^{2} & \dot{W}_{p y} \dot{W}_{p z} & \dot{W}_{p y} \dot{W}_{p x} & \boldsymbol{0}_{1 \times 7} \\ \boldsymbol{0}_{1 \times 7} & \boldsymbol{0}_{1 \times 7} & 1 & \dot{W}_{p z} & \dot{W}_{p x} & \dot{W}_{p y} & \dot{W}_{p z}^{2} & \dot{W}_{p z} \dot{W}_{p x} & \dot{W}_{p z} \dot{W}_{p y}\end{array}\right] ;$

$\boldsymbol{0}_{1 \times 7}$ : The 1-by-7 matrix of which every element is 0 ;

$D_{a}=\left[\begin{array}{lllllllllllllllllllll}K_{a 0 x} & K_{a 1 x} & K_{a 1 x y} & K_{a 1 x z} & K_{a 2 x} & K_{a 2 x y} & K_{a 2 x z} & K_{a 0 y} & K_{a 1 y} & K_{a 1 y z} & K_{a 1 y x} & K_{a 2 y} & K_{a 2 y z} & K_{a 2 y x} & K_{a 0 z} & K_{a 1 z} & K_{a 12 x} & K_{a 1 y y} & K_{a 22} & K_{a 22 x} & K_{a 2 z y}\end{array}\right]^{T}$;

The specific force error caused by the instrumentation error can be written as:

$\delta \dot{\boldsymbol{W}}=\dot{\boldsymbol{W}}_{p}-\dot{\boldsymbol{W}}_{a}=\dot{\boldsymbol{W}}_{p}-\boldsymbol{M}_{3}\left(\varphi_{z}\right) \boldsymbol{M}_{2}\left(\varphi_{y}\right) \boldsymbol{M}_{1}\left(\varphi_{x}\right)\left(\dot{\boldsymbol{W}}_{p}-\Delta f\right)$

Where:

$\delta \dot{W}$ : The specific force error;

$\dot{W}_{p}$ : The specific force measured by the accelerometer on the platform;

$\dot{\boldsymbol{W}}_{a}$ : The true specific force without any error;

$\boldsymbol{M}_{3}(\cdot) 、 \boldsymbol{M}_{2}(\cdot), \boldsymbol{M}_{1}(\cdot)$ : The coordinate transformation matrices caused by the rotation about the $\mathrm{x} 、 \mathrm{y}$ and $\mathrm{z}$ axis;

Because of the bias angles of the gyros are small enough, so $\boldsymbol{M}_{3}\left(\varphi_{z}\right) \boldsymbol{M}_{2}\left(\varphi_{y}\right) \boldsymbol{M}_{1}\left(\varphi_{x}\right)$ can be written as:

$$
\boldsymbol{I}-(\boldsymbol{\varphi} \times)=\left[\begin{array}{ccc}
1 & \varphi_{z} & -\varphi_{y} \\
-\varphi_{z} & 1 & \varphi_{x} \\
\varphi_{\mathrm{y}} & -\varphi_{x} & 1
\end{array}\right]
$$

Substituting Eq. (6) into Eq. (5) yields:

$\delta \dot{\boldsymbol{W}}=-\left[\begin{array}{ccc}0 & -\varphi_{z} & \varphi_{y} \\ \varphi_{z} & 0 & -\varphi_{x} \\ -\varphi_{x} & \varphi_{x} & 0\end{array}\right] \dot{\boldsymbol{W}}_{p}+\boldsymbol{\Delta}=\left[\begin{array}{ccc}0 & -\dot{W}_{p z} & \dot{W}_{p y} \\ \dot{W}_{p z} & 0 & -\dot{W}_{p x} \\ -\dot{W}_{p y} & \dot{W}_{p x} & 0\end{array}\right] \boldsymbol{\varphi}+\boldsymbol{\Delta}$

Substituting Eq. (2) and Eq. (4) into Eq. (7) yields: 


$$
\delta \dot{\boldsymbol{W}}=\boldsymbol{S}_{e} \int \boldsymbol{S}_{g} d t \cdot \boldsymbol{D}_{g}+\boldsymbol{S}_{a} \cdot \boldsymbol{D}_{a}=\boldsymbol{S}_{A} \cdot \mathbf{D}
$$

Where:

$$
\boldsymbol{S}_{e}=\left[\begin{array}{ccc}
0 & -\dot{W}_{p z} & \dot{W}_{p y} \\
\dot{W}_{p z} & 0 & -\dot{W}_{p x} \\
-\dot{W}_{p y} & \dot{W}_{p x} & 0
\end{array}\right] \quad \boldsymbol{S}_{A}=\left[\begin{array}{ll}
\boldsymbol{S}_{e} \cdot \int \boldsymbol{S}_{g} d t & \boldsymbol{S}_{a}
\end{array}\right] \quad \boldsymbol{D}=\left[\begin{array}{c}
\boldsymbol{D}_{g} \\
\boldsymbol{D}_{a}
\end{array}\right] .
$$

The instrumentation error separation model of the apparent velocity can be obtained by integrating Eq. (8):

$$
\delta v=S_{V} \cdot D
$$

Where:

$$
S_{V}=\int S_{A} d t
$$

\section{The New Algorithm Based on Wavelet Analysis and RELS}

\subsection{Introduction of Wavelet Analysis}

The wavelet analysis is a flexible tool to dispose signals. It has the character of good localization quality. At low frequency part, the wavelet has higher frequency resolution and lower time resolution. On the contrary, at high frequency part, the wavelet has lower frequency resolution and higher time resolution. And as a result of this quality, the wavelet analysis is an ideal tool to dispose signals.

De-nosing is an important application of the wavelet. A sequence of data signals with a certain length can be divided into a series of wavelet coefficients by the Mallat Algorithm (also called the Pyramid Algorithm). Redundant information of the original signals has been reduced in these wavelet coefficients. Combining with the threshold, the purpose of de-noising can be achieved.

The Pyramid Algorithm is derived basing on the hypothesis that the length of the signal is infinite. But actually, all the signals are finite. So a part of the filter must be cut off to adapt to the finite sequence of signal in order to realize the wavelet decomposition. So, here comes the problem called boundary effect that there is a big error between the processed signal and the origin signal. To solve the boundary effect, the boundary extension is used to increase the length of the signal and to guarantee the accurate reconstruction of the signal. The frequently used extension methods are: ( $\mathrm{L}$ is the length of the signal)

A. Zero extension. Zero extension is the simplest method. That is considering every element beyond the boundary is zero. This is equivalent to truncation of the wavelet filter.

$\tilde{c}=\{\underbrace{0, \cdots, 0}_{L}, c_{0}, c_{1}, \cdots, c_{N-1}, \underbrace{0, \cdots, 0}_{L}\}$

B. Periodical extension. This kind of extension is equivalent to connect the signal head to tail.

$$
\tilde{C}=\{\underbrace{c_{N-L}, \cdots, C_{N-2}, C_{N-1}}_{L}, c_{0}, C_{1}, \cdots, c_{N-1}, \underbrace{c_{0}, c_{1}, \cdots, c_{L-1}}_{L}\}
$$

C. Symmetrical periodic extension with boundary point as the center. This kind of extension is equivalent to fold the signal symmetrically at the boundary point.

When $\mathrm{L}$ is an even number:

$$
\tilde{C}=\{\underbrace{c_{L-1}, \cdots, c_{1}, c_{0}}_{L}, c_{0}, c_{1}, \cdots, c_{N-1}, \underbrace{c_{N-1}, c_{N-2}, \cdots, c_{N-L}}_{L}\}
$$

When $\mathrm{L}$ is an odd number: 


$$
\tilde{C}=\{\underbrace{c_{L}, \cdots, c_{2}, c_{1}}_{L}, c_{0}, c_{1}, \cdots, c_{N-1}, \underbrace{c_{N-2}, C_{N-3}, \cdots, c_{N-L-1}}_{L}\}
$$

The shortage of the zero extension is there might be a large step change if the difference between the boundary value and zero is very big. As a result, high-frequency component is introduced into this local part. When the difference between the head and tail of the signal is big, the same problem exists in the periodical extension. The symmetrical periodic extension with boundary point as the center is chosen here to avoid the periodical abrupt change and to eliminate the difference of the boundary value [4].

The main de-noising methods using wavelet are: wavelet reduction based on modulus maximum and nonlinear wavelet transformation threshold de-noising, etc. The wavelet threshold de-noising is adopted in this paper. Usually the wavelet threshold de-noising methods include: soft threshold、 hard threshold and forced wavelet de-noising:

A. The soft threshold method is comparing the wavelet coefficients with the threshold at first. Then set the wavelet coefficients which are smaller than the threshold to zero. And for those bigger than the threshold, set them to the difference with the threshold.

B. The difference of hard threshold compared with the soft threshold is when the wavelet coefficient is bigger than the threshold, it remains unchanged.

C. The forced wavelet de-noising method is setting all the high-frequency coefficients to zero compulsorily.

\subsection{Introduction of RELS}

As shown in Figure 2, $\hat{\theta}_{m} 、 \hat{\theta}_{m+1}$ are the parameter estimations at former and current moment. $P_{m} 、 P_{m+1}$ denote the covariance matrix of state estimation at former and current moment. $z(m+1)$ denotes the current measurement. $h(m+1)$ presents the current observation matrix.

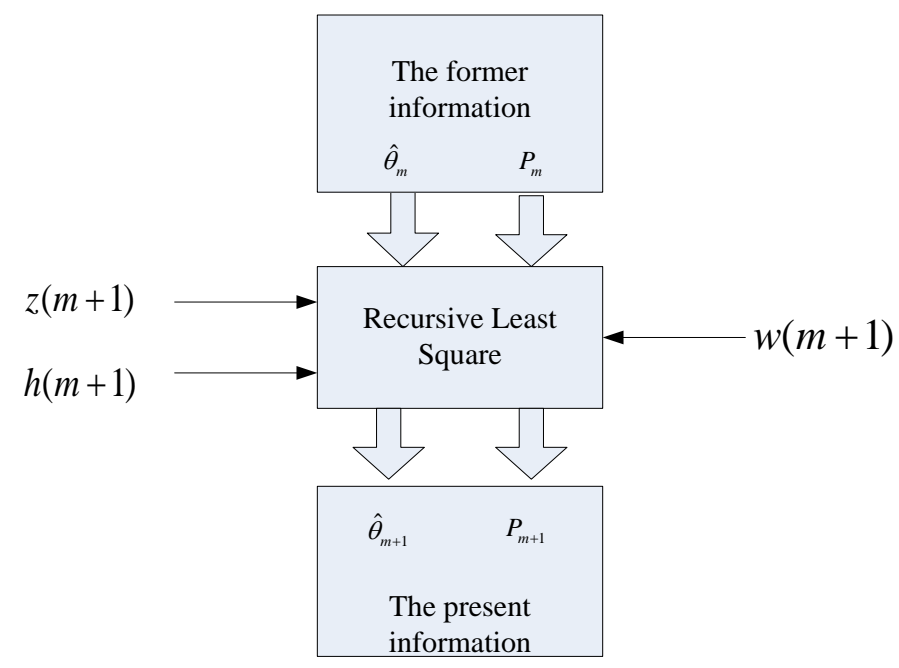

Figure 2 The information transformation of the RLS

The algorithm of the recursive extended least square is as follows:

$$
\begin{aligned}
& \hat{\boldsymbol{\theta}}_{m+1}=\hat{\boldsymbol{\theta}}_{m}+\boldsymbol{K}_{m+1}\left[z(m+1)-\boldsymbol{h}(m+1) \hat{\boldsymbol{\theta}}_{m}\right] \\
& \boldsymbol{P}_{m+1}=\boldsymbol{P}_{m}-\boldsymbol{P}_{m} \boldsymbol{h}^{T}(m+1)\left[w^{-1}(m+1)+\boldsymbol{h}(m+1) \boldsymbol{P}_{m} \boldsymbol{h}^{T}(m+1)\right]^{-1} \boldsymbol{h}(m+1) \boldsymbol{P}_{m} \\
& \boldsymbol{K}_{m+1}=\boldsymbol{P}_{m} \boldsymbol{h}^{T}(m+1)\left[w^{-1}(m+1)+\boldsymbol{h}(m+1) \boldsymbol{P}_{m} \boldsymbol{h}^{T}(m+1)\right]^{-1}
\end{aligned}
$$

Where:

$\boldsymbol{h}(m+1) \hat{\boldsymbol{\theta}}_{m}$ : The forecast of the current measurement based on the former measurement;

$z(m+1)-\boldsymbol{h}(m+1) \hat{\boldsymbol{\theta}}_{m}$ : The prediction error, also known as the new information;

$K$ : the gain matrix. 
The basic thoughts of the RLS can be concluded as [8]: the current estimation is equal to the former estimation plus the corrected items. That is the new estimation is corrected by the new measurement based on the old estimation. On the basis of RLS, the RELS takes the noise estimation to replace the noise itself. And the noise sequence is regarded as the identification object to extend the state vector.

\section{Simulation Results}

To stress the better results of the RELS, compare the results to LS. Add white noise whose standard deviation is $0.1 \mathrm{~m} / \mathrm{s}$. The initial value $P_{0}=I_{37 \times 37} \cdot 10^{6}$. Use the RELS (method 1 ) to separate the instrumentation error. The comparison of the RELS and the LS (method 2) is shown in Tab. 1.

Table 1 The comparison of two methods

\begin{tabular}{|c|c|c|c|c|}
\hline error coefficient & unit & true value & LS & RELS \\
\hline$K_{g 0 x}$ & $\circ / \mathrm{h}$ & 0.5 & -14.8795 & 0.5260 \\
\hline$K_{g 1 x}$ & $\circ / \mathrm{h} / \mathrm{g}$ & -0.1 & 0.7756 & -0.0993 \\
\hline$K_{g 1 x y}$ & $\circ / \mathrm{h} / \mathrm{g}$ & -0.01 & 5.2954 & -0.0095 \\
\hline$K_{g 1 x z}$ & $\circ / \mathrm{h} / \mathrm{g}$ & 0.02 & 741.7836 & 0.8266 \\
\hline$K_{g 2 x}$ & $\circ / \mathrm{h} / \mathrm{g}^{2}$ & $10^{-4}$ & 0.0011 & $3.2798 \times 10^{-6}$ \\
\hline$K_{g 0 y}$ & $\circ / \mathrm{h}$ & 0.3 & -0.5413 & 0.2692 \\
\hline$K_{g 1 y}$ & $\circ / \mathrm{h} / \mathrm{g}$ & 0.01 & 1.3433 & 0.0182 \\
\hline$K_{g 1 y z}$ & $\circ / \mathrm{h} / \mathrm{g}$ & 0.02 & -912.02466 & -0.8591 \\
\hline$K_{g 1 y x}$ & $\circ / \mathrm{h} / \mathrm{g}$ & -0.01 & -3.4932 & -0.0135 \\
\hline$K_{g 2 y}$ & $\circ / \mathrm{h} / \mathrm{g}^{2}$ & $10^{-4}$ & -0.0033 & $-2.9558 \times 10^{-6}$ \\
\hline$K_{g 0 z}$ & $\circ / \mathrm{h}$ & -0.5 & 0.6252 & -0.4999 \\
\hline$K_{g 1 z}$ & $\circ / \mathrm{h} / \mathrm{g}$ & 0.01 & -5.4618 & 0.0064 \\
\hline$K_{g 1 z x}$ & $\circ / \mathrm{h} / \mathrm{g}$ & -0.02 & 0.1041 & -0.0200 \\
\hline$K_{g 1 z y}$ & $\circ / \mathrm{h} / \mathrm{g}$ & 0.02 & -0.1510 & 0.0200 \\
\hline$K_{g 2 z}$ & $\circ / \mathrm{h} / \mathrm{g}^{2}$ & $10^{-4}$ & -0.0065 & $1.4449 \times 10^{-4}$ \\
\hline$K_{a 0 x}$ & $\mathrm{mg}$ & -2 & -3.4526 & -1.9999 \\
\hline$K_{a 1 x}$ & $\mathrm{mg} / \mathrm{g}$ & 0.5 & 0.57266 & 0.4997 \\
\hline$K_{a 1 x y}$ & $\mu \mathrm{g} / \mathrm{g}$ & 50 & 59.3355 & 50.0504 \\
\hline$K_{a 1 \times z}$ & $\mu \mathrm{g} / \mathrm{g}$ & 50 & $-1.6544 \times 10^{4}$ & 22.7052 \\
\hline$K_{a 2 x}$ & $\mu \mathrm{g} / \mathrm{g}^{2}$ & $3 \times 10^{-3}$ & 1.3872 & $5.2796 \times 10^{-3}$ \\
\hline$K_{a 2 x y}$ & $\mu \mathrm{g} / \mathrm{g}$ & $3 \times 10^{-3}$ & -1.6833 & $6.8857 \times 10^{-3}$ \\
\hline$K_{a 2 x z}$ & $\mu \mathrm{g} / \mathrm{g}^{2}$ & $3 \times 10^{-3}$ & $1.0720 \times 10^{3}$ & $-7.5935 \times 10^{-2}$ \\
\hline$K_{a 0 y}$ & $\mathrm{mg}$ & -2 & -7.4861 & -1.9987 \\
\hline$K_{a 1 y}$ & $\mathrm{mg} / \mathrm{g}$ & 0.5 & 1.0726 & 0.4998 \\
\hline$K_{a 1 y z}$ & $\mu \mathrm{g} / \mathrm{g}$ & 50 & $6.1692 \times 10^{3}$ & 40.5701 \\
\hline$K_{a 1 y x}$ & $\mu \mathrm{g} / \mathrm{g}$ & 50 & 637.3173 & 49.7787 \\
\hline$K_{a 2 y}$ & $\mu \mathrm{g} / \mathrm{g}^{2}$ & $3 \times 10^{-3}$ & -7.4529 & $3.4511 \times 10^{-3}$ \\
\hline$K_{a 2 y z}$ & $\mu \mathrm{g} / \mathrm{g}$ & $3 \times 10^{-3}$ & $-6.9128 \times 10^{3}$ & -0.2588 \\
\hline$K_{a 2 y x}$ & $\mu \mathrm{g} / \mathrm{g}^{2}$ & $3 \times 10^{-3}$ & -46.5305 & $-3.9891 \times 10^{-3}$ \\
\hline$K_{a 0 z}$ & $\mathrm{mg}$ & 1 & -19.9706 & 1.0557 \\
\hline$K_{a 1 z}$ & $\mathrm{mg} / \mathrm{g}$ & 0.5 & -577.1944 & 0.6839 \\
\hline$K_{a 1 z x}$ & $\mu \mathrm{g} / \mathrm{g}$ & 50 & $2.1491 \times 10^{3}$ & 44.0435 \\
\hline
\end{tabular}




\begin{tabular}{|c|c|c|c|c|}
\hline$K_{a 1 z y}$ & $\mu \mathrm{g} / \mathrm{g}$ & 50 & $7.3307 \times 10^{3}$ & 46.8627 \\
\hline$K_{a 2 z}$ & $\mu \mathrm{g} / \mathrm{g}^{2}$ & $3 \times 10^{-3}$ & $-7.4695 \times 10^{9}$ & $4.7228 \times 10^{3}$ \\
\hline$K_{a 2 z x}$ & $\mu \mathrm{g} / \mathrm{g}$ & $3 \times 10^{-3}$ & $-7.0234 \times 10^{7}$ & -5.1602 \\
\hline$K_{a 2 z y}$ & $\mu \mathrm{g} / \mathrm{g}^{2}$ & $3 \times 10^{-3}$ & $-1.0193 \times 10^{8}$ & 4.3400 \\
\hline
\end{tabular}

From Table 1, it can be seen that, most of the estimations of method 2 are very close to the true value. It is because that, the wavelet de-noising reduces the effect of the noise and the RELS makes the estimation convergence closely to the true value. The other estimations are not that good partly because the driving is not enough during the flight. For the method 1, after adding the white noise, the results totally deviate from the true values. The comparison adequately shows the merit of the proposed algorithm.

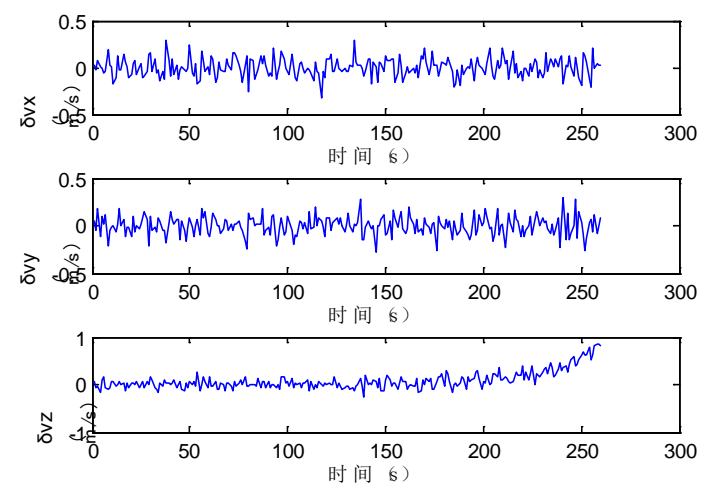

Figure 3 The residual error of method 1

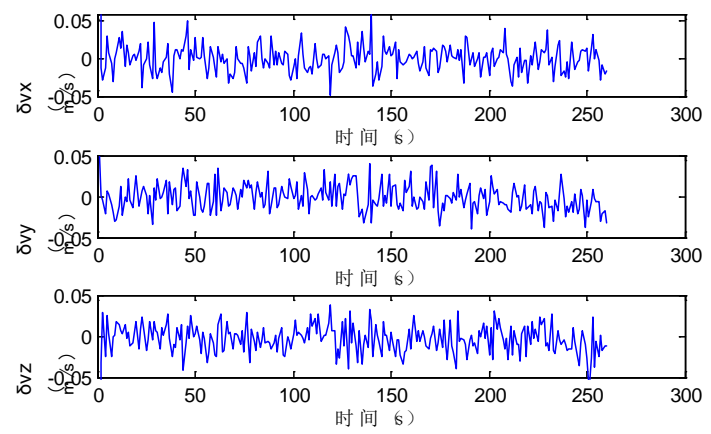

Figure 4 The residual error of method 2

The residual errors of the velocity are shown in Figure 3 and Figure 4. In Figure 3, the residual errors of velocity in $\mathrm{x} 、 \mathrm{y}$ and $\mathrm{z}$ directions are within $\pm 0.5 、 \pm 0.5, \pm 1 \mathrm{~m} / \mathrm{s}$ respectively. From Figure 4 it can be seen that, the residual errors of velocity in $x 、 y$ and $z$ directions are all within $\pm 0.05 \mathrm{~m} / \mathrm{s}$. Remarkably, the residual errors of method 2 are much smaller than method 1 . That is to say, fitting result of method 2 is more accurate. The contrast of the residual errors firmly proves the proposed algorithm is correct and effective.

\section{Conclusion}

A new algorithm is put forward creatively aiming at the shortage of the existing instrumentation error separation methods. At first, the de-noising of data is carried out by using the wavelet analysis. And then, the RELS is used to separate the error coefficients. The theoretical guidance is provided for the engineering application.

There are some advantages of the proposed algorithm. Firstly, comparing with the direct separation of the traditional methods, the noise influence has been reduced by wavelet de-noising. 
Secondly, the estimation results are closer to the true value by identifying the noise sequence and replacing the noise itself with the estimation value. So the estimation accuracy is improved a lot.

\section{References}

[1] Zhang, J.H. (1995) Evaluation and Analysis of Precision for Long-distance Rocket [M]. Published by National University of Defence Technology Press.

[2] S, Mallat and Hwang, W.L (1991) Zero-crossing of a Wavelets-transform [J], IEEE Trans. Inform. Theory, 37(4): 1019-1033.

[3] Donoho, D.L. (1995) De-nosing by soft-thresholding [J]. IEEE Transactions on Information Theory, 41(3): 613-626.

[4] Huang, Y and Cai, H. (2016) Research on real-time de-noising of floated inertial platform system FOG data [J]. Tactical Missile Technology, 2: 84-88.

[5] Ding, F and Ding, T. (2003) Performance analysis of multi-innovation stochastic gradient identification methods, J. Control Theory \& Applications, 20(6): 870-874.

[6] Liu, J.K, Shen, X.R and Zhao, L. (2013) System identification theory and Matlab simulation [M]. Beijing: Publishing House of Electronics Industry.

[7] Albert Boggess, Francis J and Narcowich. (2004) Wavelet and Fourier analysis [M]. Publishing House of Electronics Industry.

[8] Wang, D.Q. (2009) Recursive extended least squares identification method based on auxiliary models [J]. Control Theory \& Applications, 26(1): 51-56. 